\title{
16S rRNA gene-based metagenomic analysis reveals differences in bacteria-derived extracellular vesicles in the urine of pregnant and non-pregnant women
}

\author{
Jae Young Yoo ${ }^{1,2,8}$, Mina Rho ${ }^{3,8}$, Young-Ah You ${ }^{1}$, Eun Jin Kwon ${ }^{1}$, Min-Hye Kim ${ }^{4}$, Sungmin $\mathrm{Kym}^{5}$, \\ Young-Koo Jee ${ }^{6}$, Yoon-Keun $\mathrm{Kim}^{7}$ and Young Ju Kim ${ }^{1}$
}

Recent evidence has indicated that bacteria-derived extracellular vesicles (EVs) are important for host-microbe communication. The aims of the present study were to evaluate whether bacteria-derived EVs are excreted via the urinary tract and to compare the composition of bacteria-derived EVs in the urine of pregnant and non-pregnant women. Seventy-three non-pregnant and seventy-four pregnant women were enrolled from Dankook University and Ewha Womans University hospitals. DNA was extracted from urine EVs after EV isolation using the differential centrifugation method. 16S ribosomal RNA (16S rRNA) gene sequencing was performed using high-throughput 454 pyrosequencing after amplification of the V1-V3 region of the 16S rDNA. The composition of 13 taxa differed significantly between the pregnant and non-pregnant women. At the genus level, Bacillus spp. EVs were more significantly enriched in the urine of the pregnant women than in that of the non-pregnant women $(45.61 \%$ vs $0.12 \%$, respectively). However, Pseudomonas spp. EVs were more dominant in non-pregnant women than in pregnant women (13.2\% vs $4.09 \%$, respectively). Regarding the compositional difference between pregnant women with normal and preterm delivery, EVs derived from Ureaplasma spp. and the family Veillonellaceae (including Megasphaera spp.) were more abundant in the urine of preterm-delivered women than in that of women with normal deliveries. Taken together, these data showed that Bacillus spp. EVs predominate in the urine of pregnant women, whereas Pseudomonas spp. EVs predominate in the urine of non-pregnant women; this suggests that Bacillus spp. EVs might have an important role in the maintenance of pregnancy. Experimental \& Molecular Medicine (2016) 48, e208; doi:10.1038/emm.2015.110; published online 5 February 2016

\section{INTRODUCTION}

Pregnant women undergo profound anatomical, physiological and biochemical changes that are influenced by hormonal and physical fluctuations. ${ }^{1}$ Pregnancy is associated with an increase in cervical secretions and a decrease in vaginal $\mathrm{pH}$, which has long been known to reflect increased levels of Lactobacillus acidophilus. ${ }^{1}$ Microbial studies can help to explain differences in cytokine levels (in the vagina and amniotic fluids), variations in the $\mathrm{pH}$ of such fluids and the differential susceptibility to infections in pregnant and non-pregnant women. ${ }^{1}$ Each year, $\sim 15$ million babies are born prematurely; this number constitutes $\sim 10 \%$ of all infants. ${ }^{2}$ Established preterm birth (PTB) risk factors include infections (of the urinary tract and bacterial vaginosis), uterine and cervical anomalies, in vitro fertilization, inadequate maternal nutrition and genetic factors. ${ }^{3}$ Many researchers have suggested that intrauterine infections could explain $25-40 \%$ of PTBs. ${ }^{4,5}$ In addition, pregnant women should have asymptomatic bacteriuria screening with each trimester of pregnancy and should be treated, if positive, to reduce the risk of recurrent urinary tract infection and preterm labor. ${ }^{6,7}$

\footnotetext{
${ }^{1}$ Department of Obstetrics and Gynecology, School of Medicine and Ewha Medical Institute, Ewha Womans University, Ewha Medical Center, Seoul, South Korea; ${ }^{2}$ National Agency for Breeding Stock Improvement, National Institute of Animal Science, Cheonan, Korea; ${ }^{3}$ Department of Computer Engineering, Hanyang University, Seoul, South Korea; ${ }^{4}$ Department of Internal Medicine, School of Medicine, Ewha Womans University, Seoul, South Korea; ${ }^{5}$ Department of Internal Medicine, Inje University College of Medicine, Busan, South Korea; ${ }^{6}$ Department of Internal Medicine, College of Medicine, Dankook University, Cheonan, South Korea and 'Department of Medicine, School of Medicine, Ewha Womans University and Ewha Institute of Convergence Medicine, Ewha Medical Center, Seoul, South Korea

${ }^{8}$ These authors contributed equally to this work.

Correspondence: Dr YK Kim, Department of Medicine, School of Medicine, Ewha Womans University and Ewha Institute of Convergence Medicine, Ewha Medical Center, 1071, Anyangcheon-ro, Yangcheon-gu, Seoul 158-710, South Korea.

E-mail: juinea@ewha.ac.kr

or Dr YJ Kim, Department of Obstetrics and Gynecology, School of Medicine and Ewha Medical Institute, Ewha Womans University, Ewha Medical Center, 1071, Anyangcheon-ro, Yangcheon-gu, Seoul 158-710, South Korea.

E-mail: kkyj@ewha.ac.kr
}

Received 29 June 2015; revised 7 October 2015; accepted 8 October 2015 
Bacteria constitutively secrete extracellular vesicles (EVs) into the extracellular milieu. ${ }^{89}$ The EVs are evolutionarily conserved nanoparticles that have an important biological function in intercellular communication. Gram-negative bacteria-derived EVs, or outer membrane vesicles, are spherical, nanometer-sized bilayered proteolipids, called nanovesicles, that not only harbor endotoxins (lipopolysaccharides) but also virulence proteins, and bacterial DNA and RNA. ${ }^{8}$ In addition, Gram-positive bacteria were found to secrete EVs, which harbor bacterial cell wall components, including peptidoglycan and lipoteichoic acid, and also virulence proteins and nucleic acids. ${ }^{10}$ Recent evidence has shown that bacteria-derived EVs might be an etiological agent for the development of inflammatory diseases that were once believed to be noninfectious. ${ }^{11}$ With regard to the distribution of Gram-negative bacterial outer membrane vesicles, when they were injected intraperitoneally, the outer membrane vesicles were found to spread to the entire mouse body and accumulate in the liver, lung, spleen and kidney within $3 \mathrm{~h}$ of administration. ${ }^{12}$ In addition, the EVs had been isolated from amniotic fluid and urine. ${ }^{13,14}$

Metagenomics is the study of genetic material that is recovered directly from environmental samples, including urine. While traditional microbiology relies on microbial genes or the genomes of cultivated clonal cultures, metagenomic sequencing is performed using the cloning of specific genes, often the 16S ribosomal RNA (16S rRNA) gene or whole genome from a natural sample. ${ }^{1,15}$ The first metagenomic studies conducted using high-throughput sequencing used massively parallel 454 pyrosequencing. ${ }^{16}$ In pregnant women, metagenomics were used to explore the bacterial composition of stool and vaginal and amniotic fluid. ${ }^{1,15,17,18}$ In addition, the bacteria of pregnant women was associated with poor outcomes for both the mother and child, including PTB and low birth weight. ${ }^{7,19}$ However, bacteria-derived EV composition in the urine of normal or pregnant women (including PTB) has not yet been established.

The purpose of the present study was to evaluate whether bacteria-derived EVs are excreted via the urinary tract, and if so, to compare the features of such EVs in the urine of pregnant and non-pregnant women.

\section{MATERIALS AND METHODS}

\section{Participants and sample collection}

In total, 73 non-pregnant women and 74 pregnant women (39 had normal deliveries; 35 had preterm deliveries) were enrolled from Dankook University and Ewha Womans University Medical Center, respectively, from 2006to 2008. We recruited women who delivered preterm infants ( $<37$ weeks of gestation) at our hospital. Members of the normal delivery group (who underwent term delivery at $\geqslant 37$ weeks of gestation) were selected from women who had undergone prenatal examinations at our hospital and were followed up until delivery. The inclusion criteria were a singleton birth and a gestational age of 25-42 weeks. We excluded women who had multiple births, stillbirths, infants with congenital anomalies, chronic hypertension, placenta previa and abruption placenta. The control (non-pregnant) group was selected from women who underwent routine health examinations at Dankook University Hospital.

The present study was approved by the Institutional Review Board of Ewha Womans University Hospital (ECT 127-07) and Dankook University Hospital (DKUH IRB 2013-04-0125). The methods were conducted in accordance with the approved guidelines, and informed consent was obtained from all of the subjects.

\section{Data collection and measurement}

Trained interviewers collected general epidemiological and clinical data from the participants, who signed consent forms before enrollment. The weight and height were measured, and blood samples were obtained using standard protocols. The body mass index was calculated as the weight divided by the height squared $\left(\mathrm{kg} \mathrm{m}^{-2}\right)$. Blood samples were collected from the median cubital vein into Vacutainer tubes that contained EDTA or serum tubes (BD, Franklin Lakes, NJ, USA), after overnight fasting. The glucose concentration was determined using L-Type Glu2 Reagent Kits (Wako, Osaka, Japan). The cholesterol concentration was determined using L-type CHO M Reagent Kits (Wako). The AST (aminotransaminase) and ALT (alanine aminotransaminase) concentrations were determined using Pureauto S AST-L Reagent Kits (Sekisui Medical, Tokyo, Japan). The HDL (high-density lipoprotein) concentration was determined using Cholestest N HDL Reagent Kits (Sekisui Medical). The creatinine concentration was determined using Clinimate CRE Reagent Kits (Sekisui Medical). Measurements were recorded with the aid of an automatic analyzer (Model 7180; Hitachi, Tokyo, Japan). The white blood cell counts and hemoglobin levels were determined using STROM.-FB, Cellpack, Cell Sheath Reagent Kits and the hematology analyzer Sysmex XE-2100 (TOA Medical Electronics, Kobe, Japan). Midstream clean-catch urine samples were collected in sterile urine bottles when the subjects were admitted to the hospital before delivery.

EV isolation and DNA extraction from human urine samples EVs in human urine were isolated using the differential centrifugation method as described previously. ${ }^{20}$ For the extraction of DNA in isolated EVs, $1 \mu \mathrm{g}$ (based on the protein amount) of the EVs was boiled at $100{ }^{\circ} \mathrm{C}$ for $15 \mathrm{~min}$, and then it was centrifuged at $10000 \mathrm{~g}$ for $20 \mathrm{~min}$. The quality and quantity of the DNA were measured using the NanoDrop assay.

\section{Emulsion-based PCR for metagenomic sequencing}

Sequencing was performed by Macrogen (Seoul, South Korea) using a GS-FLX Titanium Sequencer System (Roche, Basel, Switzerland). Briefly, genomic DNA was amplified from urine by the polymerase chain reaction (PCR). The method used a $16 \mathrm{~S}$ rDNA fusion primer (27F, 5'-GAGTTTGATCMTGGCTCAG-3' and the primer 518R, 5'-WTTACCGCGGCTGCTGG-3') to amplify the V1-V3 region. A FastStart High Fidelity PCR System was used (Roche, Basel, Switzerland). Amplification was performed on emulsions that were formed by mixing oil and amplicon solutions with vigorous shaking. The Tissue Lyser II System (Qiagen, Hilden, Germany) and GS-FLX-plus GSFLX-plus-Emulsion-Based PCR Kit (454 Life Sciences, Branford, CT USA) were used to create microreactors that contained both an amplification mix and a single bead. The emulsions were dispensed into a 96-well plate, and a PCR amplification program was run according to the manufacturer's recommendations. A 20-ng aliquot of each DNA sample was included in each $50-\mu$ PCR reaction mix. The PCR proceeded at $94^{\circ} \mathrm{C}$ for $3 \mathrm{~min}$, followed by 35 cycles of $94^{\circ} \mathrm{C}$ for 
Table 1 Basic characteristics of the study subjects

\begin{tabular}{|c|c|c|c|c|}
\hline & \multirow[b]{2}{*}{ Non-pregnant women $(n=73)$} & \multicolumn{2}{|c|}{ Pregnant women } & \multirow[b]{2}{*}{ P-value ${ }^{a}$} \\
\hline & & Normal $(n=39)$ & Preterm $(\mathrm{n}=35)$ & \\
\hline Age (years) & $32.2(12.3)$ & 31.96 (3.99) & $33.11(3.75)$ & $0.797^{b}$ \\
\hline Gestational weeks & & $39.73(1.88)$ & $33.57(2.76)$ & 0.000 \\
\hline Nulliparity (n, \%) & & $28(59.57)$ & $15(41.67)$ & $0.106^{c}$ \\
\hline \multicolumn{5}{|l|}{ Birth outcome } \\
\hline Birth weight (g) & & $3342.13(314.80)$ & $2179.17(624.21)$ & 0.000 \\
\hline AS $1 \mathrm{~min}$ & & $9.66(0.700)$ & $7.67(2.12)$ & 0.000 \\
\hline AS $5 \mathrm{~min}$ & & $9.98(0.15)$ & $8.86(1.57)$ & 0.000 \\
\hline IUGR ( $n, \%)$ & & $0(0.00)$ & $4(2.78)$ & - \\
\hline
\end{tabular}

Abbreviations: ANOVA, analysis of variance; AS, Apgar score, IUGR, intrauterine growth restriction.

Numerical data are presented as the means with s.d.s.

a Statistical $P$-values were derived by comparing the normal and preterm group data using a $t$-test.

bStatistical $P$-values were derived by comparing the control, normal and preterm group data using ANOVA.

'Statistical $P$-values were derived by comparing normal and preterm group data using a $\chi^{2}$ test.

$15 \mathrm{~s}, 55^{\circ} \mathrm{C}$ for $45 \mathrm{~s}$ and $72{ }^{\circ} \mathrm{C}$ for $1 \mathrm{~min}$, and a final elongation step at $72^{\circ} \mathrm{C}$ for $8 \mathrm{~min}$. After emulsion-based PCR amplification, the amplicons were purified using an AMpure Bead Kit (Beckman Coulter, Brea, CA, USA), and the DNA levels were quantified using the Picogreen method (Invitrogen, Carlsbad, CA, USA). Next, the amplicons were diluted, pooled and analyzed using a GS-FLX Titanium Sequencer (Roche, Basel, Switzerland) according to the manufacturer's instructions.

\section{Next-generation sequencing using the Roche 454 Genome Sequencer FLX Titanium platform}

After PCR amplification, each emulsion was chemically broken, and the beads that carried amplified DNA libraries were recovered and washed via filtration. DNA-positive beads were purified using the biotinylated primer A (complementary to adaptor A), which binds to streptavidin-coated magnetic beads. Beads that had DNA libraries were then separated from the magnetic beads by melting the doublestranded amplification products, thus creating a population of beadbound single-stranded template DNA fragments. The sequencing primer was then annealed to the amplified single-stranded DNAs. Finally, the beads that had such DNA were counted using a particle counter (Beckman Coulter). Sequencing was performed using the Roche 454 Genome Sequencer FLX Titanium platform (Life Sciences), and each sample was loaded into a single region of a $70-$ to $75-\mathrm{mm}$ Roche 454 PicoTiter plate (Life Sciences) that was fitted with an eight-lane gasket.

\section{Selection of 16S rRNAs and taxonomic assignments}

Sequencing reads of high quality were retained after checking both the score quality (average Phred score $>20$ ) and read lengths (>300 bp). Operational taxonomic units (OTUs) were defined using UCLUST and USEARCH; ${ }^{21}$ taxonomic assignment was achieved using QIIME ${ }^{22}$ against the 16S rRNA sequence database of GreenGenes 8.15.13. ${ }^{23}$ Based on the identified similarities, all of the 16S rRNA sequences were assigned at the following taxonomic levels: species, $>97 \%$ similarity; genus, $>94 \%$ similarity; family, $>90 \%$ similarity; order, $>85 \%$ similarity; class, $>80 \%$ similarity; and phylum, $>75 \%$ similarity. Bacterial compositions at the genus level were plotted as heat maps if significant between-group differences were evident (greater than a twofold difference between the pregnant and nonpregnant women).

\section{Statistical analysis}

The results are presented as the mean \pm s.d. The basic characteristics, including the age, maternal features and birth outcomes, were compared using a one-way analysis of variance or $t$-test. In addition, the clustering characteristics were compared using the $\chi^{2}$ test or $t$-test. Statistical analyses were performed with the aid of SAS software (Version 9.3; SAS Institute, Cary, NC, USA). In addition, hierarchical clustering in the $\mathrm{R}$ package was applied to identify the groups of samples that have similar bacterial composition. Statistical significance was considered to be present when the probability value $(P)$ was $<0.05$.

\section{RESULTS}

\section{General characteristics of the study subjects}

Table 1 shows the characteristics of all of the study subjects. When the normal and preterm delivery groups were compared, the gestational weeks were significantly different (39.73 vs 33.57 weeks, $P<0.001)$. The birth weight was significantly lower in the preterm group ( 3342.13 vs $2179.17 \mathrm{~g}, P<0.001)$. In addition, the infants who underwent preterm delivery had significantly lower Apgar scores (at both 1 and $5 \mathrm{~min}$ ) compared with the infants who were delivered normally $(P<0.001)$. However, no significant difference in the maternal age was evident among the groups $(P>0.05)$.

\section{Differences in bacteria-derived EVs in urine by pregnancy status}

Hierarchical clustering was performed at the genus level on the bacterial groups that exhibited level changes that were $>2$-fold between the pregnant and non-pregnant women, but any taxon whose average abundance (composition) was below $1 \%$ in the enriched group was excluded from this heatmap (Figure 1). We performed 20 taxa for hierarchical clustering. As shown in 


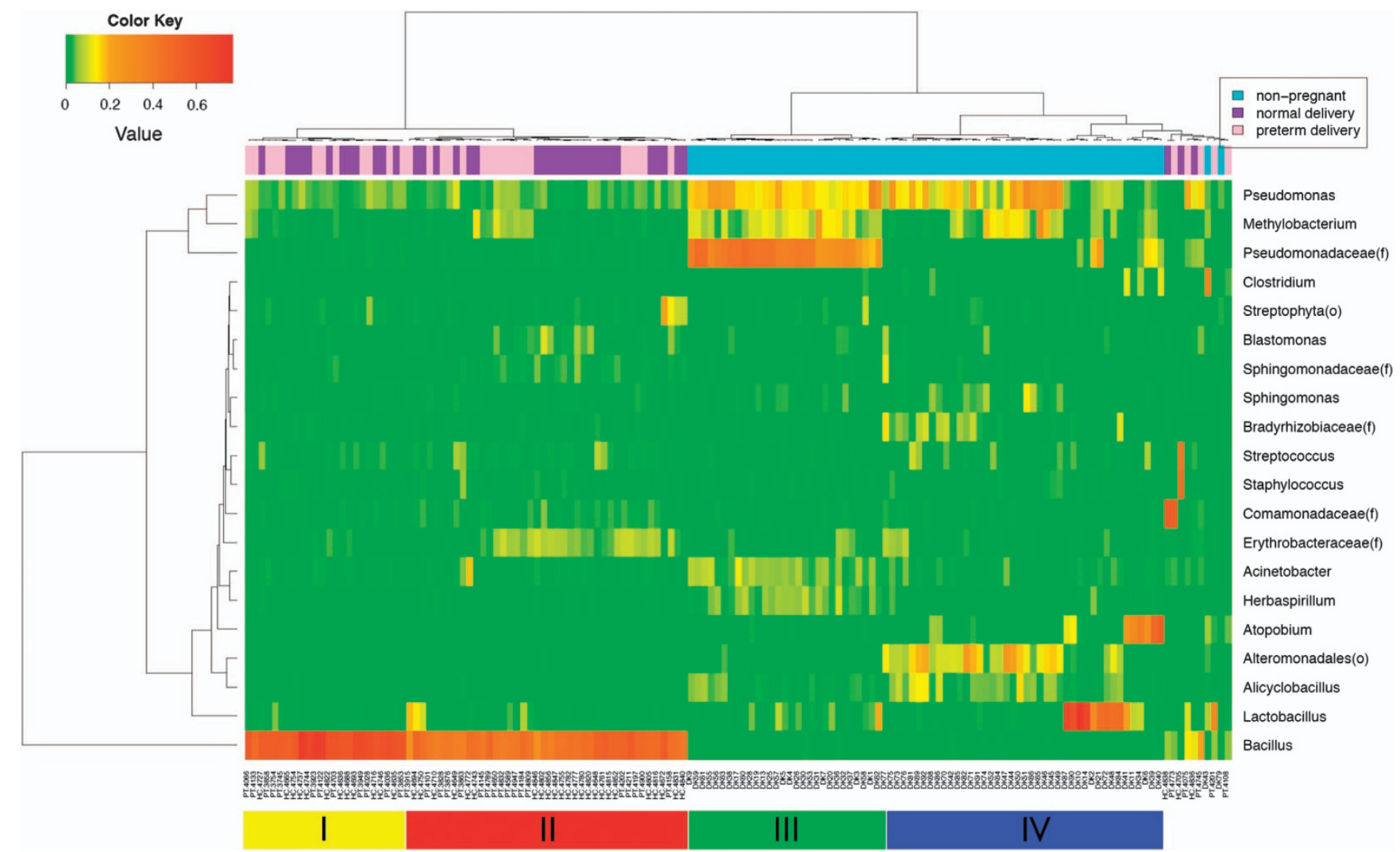

Figure 1 Abundant taxa yielding bacteria-derived extracellular vesicles in the urine of pregnant women (yellow and red bars) and nonpregnant women (green and blue bars). Right: 20 taxa that (i) exhibited greater than twofold changes in abundance between the pregnant and non-pregnant women, and (ii) constituted $>1 \%$ of the average composition in the enriched group. Genus names are used to identify the taxa. The taxa without generic names are annotated by either "f" (=family name) or "o" (=order name). The phenotypic differences among the clusters are summarized in Supplementary Table 2 (Class I subjects are under the yellow bar, and Class II subjects are under the red bar) and Supplementary Table 3 (Class III subjects are under the green bar, and Class IV subjects are under the blue bar). Each cell in the heatmap is the bacterial relative proportion in a sample. For each sample listed on the $x$ axis, the relative proportion of the bacteria (listed vertically along the $y$ axis on the right side of the plot) is represented by a color: green corresponds to low abundance, and red corresponds to high abundance. See the color key legend at the top left corner.

Figure 1, hierarchical clustering that used the $\mathrm{R}$ package was applied to identify groups of samples that had similar bacterial compositions. The samples were segregated into two groups. The first group was mainly composed of microbes that were present in the samples from pregnant women, and the second group was principally composed of microbes that were present in the samples from non-pregnant women. The first group was included in two clusters (Class I and Class II), and the second group was included in three clusters (Class III, Class IV and other cluster samples). The pregnant women principally harbored bacteria and EVs of Bacillus spp. In addition, clustering a total of 1863738 sequences resulted in 3762 OTUs using the threshold of $97 \%$ sequence similarity. The read numbers were identified using the analysis of urine metagenomic data by next-generation sequencing (Supplementary Table 1).

Supplementary Figure 1 and Table 2 show abundant bacterial genera in pregnant and non-pregnant women. When pregnant and non-pregnant women were compared, the levels of 13 microbial taxa exhibited significant differences $(P<0.05)$. In particular, Bacillus spp. was the taxon that was more significantly enriched in pregnant women (mean composition $=45.61 \%)$ than in non-pregnant women (mean composition $=0.12 \%)(P<0.001)$. However, Pseudomonas spp. was the taxon that was more enriched in non-pregnant women (mean composition $=14.23 \%)$ than in pregnant women (mean composition $=4.09 \%)(P<0.001)$. An operational taxonomic unit assigned to the family Pseudomonadaceae was also significantly enriched in non-pregnant women $(P<0.001)$. Lactobacillus spp. was significantly enriched in non-pregnant women $(8.44 \%$ vs $1.61 \%, P<0.001)$.

The characteristics of pregnant (Supplementary Table 2) and non-pregnant women (Supplementary Table 3) were analyzed by hierarchical clustering. In pregnant women, no characteristics significantly differed $(P>0.05)$. Class I and II are expressed as clusters of yellow and red bars, respectively, in Figure 1 (Supplementary Table 2). In pregnant women, all of the characteristics were not significantly different $(P>0.05)$. 
Class III and IV were expressed as clusters of green and blue bars, respectively, in Figure 1 (Supplementary Table 3). The age was significantly lower in Class I compared with that in Class II (28.14 vs $34.88, P<0.05)$ (Supplementary Table 3 ). However, other characteristics of the subjects were not significantly different $(P>0.05)$.
Compositional difference of bacteria-derived EVs in the urine from pregnant women who underwent normal vs preterm delivery

Figure 2 and Supplementary Figure 2 show the abundant bacterial genera in women who underwent normal and preterm delivery. The bacteria-derived EVs were similarly

Table 2 Abundant bacteria-derived EVs in the urine of pregnant women and non-pregnant controls

\begin{tabular}{|c|c|c|c|c|}
\hline Taxon & Mean of non-pregnant women (\%) & Mean of pregnancy (\%) & Fold change & $\mathrm{P}$-value $\mathrm{a}^{\mathrm{a}}$ \\
\hline Erythrobacteraceae(f) & $0.9847 \pm 0.0206$ & $2.8276 \pm 0.0330$ & 2.8716 & $8.47 E-05$ \\
\hline Sphingomonas & $1.5396 \pm 0.0236$ & $0.6007 \pm \pm 0.0067$ & 0.3902 & $1.57 \mathrm{E}-03$ \\
\hline Pseudomonas & $14.2252 \pm 0.0797$ & $4.0889 \pm 0.0327$ & 0.2874 & $1.21 \mathrm{E}-16$ \\
\hline Bradyrhizobiaceae(f) & $1.5951 \pm 0.0324$ & $0.4560 \pm 0.0061$ & 0.2859 & $4.14 E-03$ \\
\hline Lactobacillus & $8.4385 \pm 0.1855$ & $1.6068 \pm 0.0480$ & 0.1904 & $3.11 E-03$ \\
\hline Herbaspirillum & $1.9899 \pm 0.0272$ & $0.1409 \pm 0.0040$ & 0.0708 & $1.77 \mathrm{E}-07$ \\
\hline Pseudomonadaceae(f) & $15.4593 \pm 0.1797$ & $0.6549 \pm 0.0114$ & 0.0424 & $9.58 \mathrm{E}-10$ \\
\hline Atopobium & $3.8597 \pm 0.1090$ & $0.1017 \pm 0.0062$ & 0.0264 & $4.39 E-03$ \\
\hline Alicyclobacillus & $3.1655 \pm 0.0351$ & $0.0325 \pm 0.0020$ & 0.0103 & $7.55 E-11$ \\
\hline Alteromonadales(o) & $5.4748 \pm 0.0726$ & $0.0022 \pm 0.0002$ & 0.0004 & $1.16 \mathrm{E}-08$ \\
\hline
\end{tabular}

Percentage data are presented as numbers with s.d.s. The taxa are shown at the genus level; those that lacked a genus name are annotated by either "f" (=family name) or "o" (= order name).

a Statistical $P$-values were calculated by comparing data from pregnant and non-pregnant women using the $t$-test.

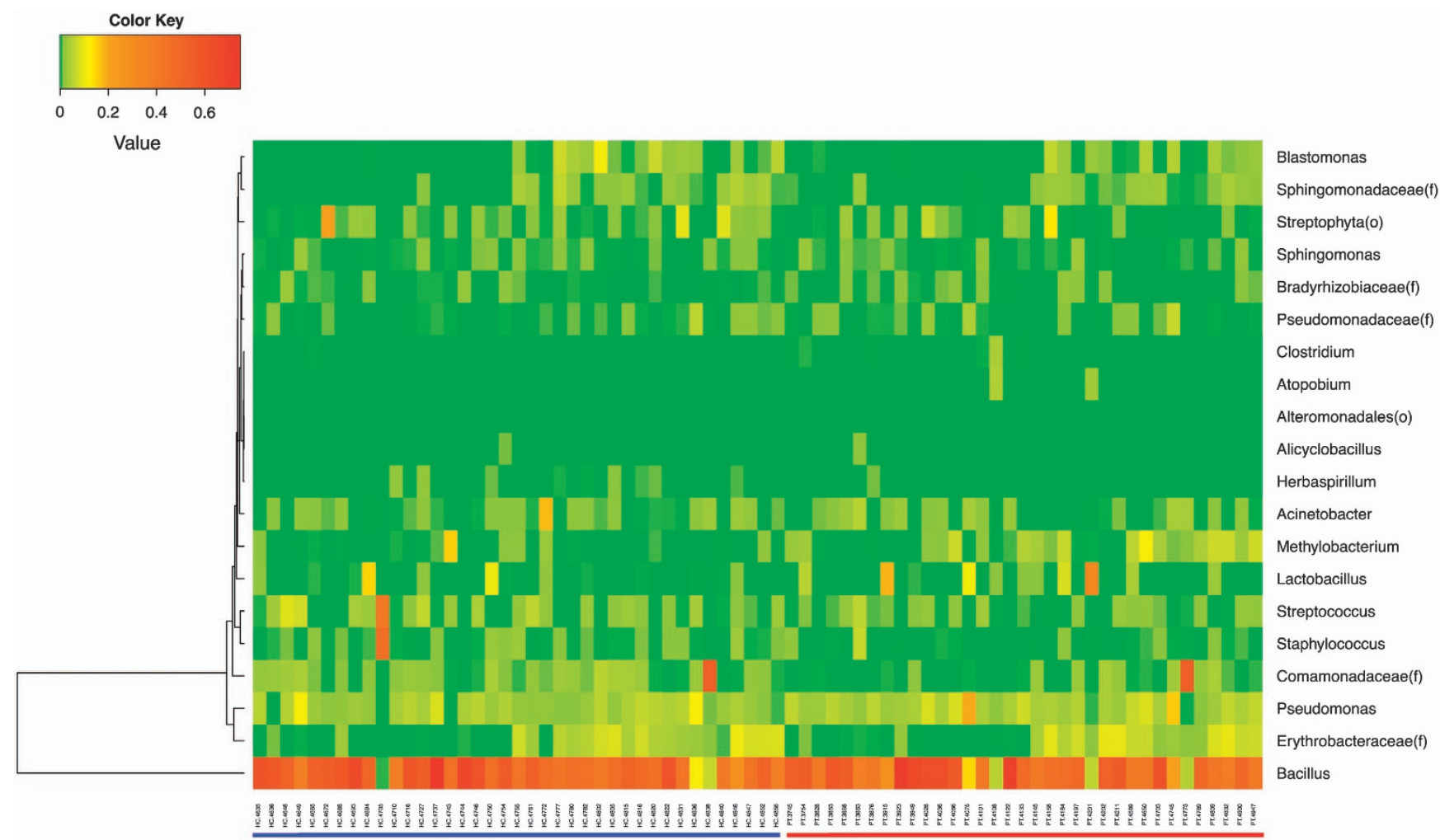

Figure 2 Abundant taxa that yielded bacteria-derived extracellular vesicles in the urine of women who experienced normal (blue bar) and preterm (red bar) delivery. Right: taxa that (i) showed greater than twofold changes in abundance between normal and preterm delivery, and (ii) constituted $>1 \%$ average microbial composition in the enriched group. Genus names are used to identify the taxa. The taxa without generic names are annotated by either "f" (=family name) or "o" (= order name). 
Table 3 Case descriptions of subjects that yielded positive urinary metagenomic results

\begin{tabular}{|c|c|c|c|c|c|c|c|c|}
\hline & $\begin{array}{c}\text { Maternal age } \\
\text { (years) }\end{array}$ & Gravida/para & $\begin{array}{l}\text { Delivery } \\
\text { (weeks) }\end{array}$ & $\begin{array}{c}\text { Birth weight } \\
\text { (g) }\end{array}$ & AS $1 \mathrm{~min}$ & AS $5 \mathrm{~min}$ & Neonatal outcomes & $\begin{array}{l}\text { Name of taxa from } \\
\text { urine metagenome }\end{array}$ \\
\hline Case 1 & 41 & $6 / 1$ & 30.4 & 1530 & 5 & 7 & $\begin{array}{l}\text { Pneumomediastinum } \\
\text { HMD } \\
\text { GMH }\end{array}$ & $\begin{array}{l}\text { Lactobacillus } \\
\text { Ureaplasma } \\
\text { Megasphaera } \\
\text { Atopobium }\end{array}$ \\
\hline
\end{tabular}

Abbreviations: AS, Apgar score; GHM, germinal matrix hemorrhage; HMD, hyaline membrane disease; RDS, respiratory distress syndrome; UTI, urinary tract infection.

distributed between normal and preterm delivery women. In addition, the phyla Firmicutes (family Veillonellaceae) and Tenericutes (Ureaplasma spp.) were rarely detected in pregnant women. Interestingly, Ureaplasma spp. was enriched in only one case from the preterm delivery women. In addition, family Veillonellaceae was shown in two cases from the preterm delivery women (Supplementary Figure 3).

Thus, we focused on the two cases of preterm delivery women that showed the abundant bacteria-derived EVs with Ureaplasma spp. and family Veillonellaceae. Table 3 shows the abundant bacteria genera in pregnant women who gave PTB; urinary metagenomic data were used to derive these values. In case 1 , a gravida 6 , para 1 pregnant woman was delivered via cesarean section because of spontaneous preterm labor and premature rupture of the membrane. One pregnant woman underwent the McDonald operation at 27 weeks of gestation because her cervical length was short. The baby's birth weight was $1530 \mathrm{~g}$, and theApgar scores were $5(1 \mathrm{~min})$ and 7 ( $5 \mathrm{~min})$. EVs derived from several bacteria, including Lactobacillus spp., Ureaplasma spp., Atopobium spp. and Megasphaera spp., were evident upon urinary metagenomic analysis. The infant had a pneumomediastinum, hyaline membrane disease and a germinal matrix hemorrhage. In case 2, a gravida 3, para 2 pregnant woman delivered an infant normally after preterm labor. The birth weight was $1310 \mathrm{~g}$, and the Apgar scores were 7 ( $1 \mathrm{~min})$ and $9(5 \mathrm{~min})$. EVs derived from several bacteria, including the Fusobacterium spp., Megasphaera spp., Atopobium spp., Prevotella spp. and Sneathia spp., were detected. The infant had meningitis, a urinary tract infection, respiratory distress syndrome, lenticulostriate vasculopathy and congenital pneumonia.

\section{DISCUSSION}

In many human microbiome studies, including the Human Microbiome Project, the bacterial compositions of various body sites were analyzed via $16 \mathrm{~S}$ rRNA sequencing; such sequences serve as ubiquitous markers of bacterial genomes. ${ }^{24}$ Streptococcus, Bacteroides and Lactobacillus are the major genera in oral, gut and vaginal samples from normal subjects. ${ }^{25}$
In addition, pregnancy was associated with the dominance of Lactobacillus spp. ${ }^{15}$ Recent evidence has indicated that bacteria-derived EVs have key roles in the intercellular communication between the host and commensal microbes, in terms of both normal physiological and pathogenic processes. ${ }^{26}$ Metagenomics is the study of genetic material that is taken directly from environmental samples. Recent studies use whole genome or $16 \mathrm{~S}$ rRNA-based sequencing to obtain the information in the microbiome. Initially, $16 \mathrm{~S}$ rRNA-based gene sequencing is cost-effective for identifying the composition of bacteria or bacteria-derived vesicles, ${ }^{27}$ although the $16 \mathrm{~S}$ rRNAbased approaches were known to be limited by the short read lengths obtained, sequencing errors and difficulties in assessing OTUs. ${ }^{28}$ In the present study, we sequenced the V1-V3 region of the 16S rRNA gene to explore the composition of bacteriaderived EVs in the urine of pregnant and non-pregnant women. The abundance levels of 13 genera differed significantly when the urine of pregnant and non-pregnant women was compared. In particular, Bacillus spp. was the most enriched EV taxon in pregnant women, whereas Pseudomonas spp. was the most enriched in non-pregnant women. In addition, the levels of EVs from Ureaplasma spp. and the family Veillonellaceae (including Megasphaera spp.) were higher in the urine of pregnant women who underwent preterm delivery than in those who experienced normal delivery.

Bacterial transmission from mother to fetus occurs regularly during human pregnancy. ${ }^{29}$ Gram-positive bacteria, such as Bacillus spp., have been isolated principally from the urine of pregnant women. ${ }^{30}$ Recently, our group revealed that Grampositive bacteria secrete EVs. ${ }^{12}$ In the present study, we found that bacteria-derived EVs, injected intramuscularly, are excreted partly via the urinary tract and that EVs derived from Bacillus spp. are dominant during pregnancy (compared with non-pregnancy). Bacillus spp. is a heterogeneous group of Gram-positive, facultative, anaerobic endospore-forming bacteria. $^{31}$ The genus Bacillus, especially Bacillus. subtilis, is usually found in the gut and in fermented foods, where they cooperate with other microorganisms during fermentation. ${ }^{32}$ A principal characteristic of Bacillus spp. is the ability to 
produce a wide range of anti-microbial compounds that are active against both bacteria and fungi. ${ }^{31}$ Taken together, these data suggest that EVs derived from Bacillus spp. might have an important role in the maintenance of pregnancy.

Some evidence has shown that low birth weight infants born after preterm premature rupture of the membranes are heavily infected with Pseudomonas spp. ${ }^{33}$ Pseudomonas aeruginosa is seldom isolated from the urine of pregnant women. ${ }^{30}$ Changes in natural killer cell numbers, phenotypes and activities occur during pregnancy, which suggests that such cells are hormonally regulated. ${ }^{34}$ Immunological changes afford some self-defense benefits; the fetus is protected from infectious agents such as bacteria. ${ }^{35}$ In addition, the levels of $P$. aeruginosa are positively associated with those of interleukin- $8 .^{36}$ In addition, genitourinary infections in pregnant women were found to significantly increase the levels of interleukin- 8 in the urine. ${ }^{37}$ Interestingly, the present study showed that EVs derived from the family Pseudomonadaceae, especially Pseudomonas spp., were significantly enriched in the urine of non-pregnant women compared with pregnant women. Collectively, these data suggest that EVs derived from Pseudomonas spp. might be important in the maintenance of the non-pregnant state.

The present study showed that EVs derived from Ureaplasma spp. were more frequently detected in the urine of women who underwent preterm delivery compared with those who underwent normal delivery. In addition, this study showed that the composition of EVs derived from Ureaplasma spp., Fusobacterium spp. and Sneathia spp. was increased in the urine of preterm-delivered women with premature babies who had pneumonia, meningitis or urinary tract infections. Ureaplasma urealyticum, Sneathia spp. and Fusobacterium spp. are bacteria that are commonly isolated from the amniotic cavity of women who enter preterm labor and experience preterm premature rupture of the membrane. ${ }^{5,38}$ In particular, Ureaplasma is a common pathogen of the urogenital tract, and such bacteria are recognized as important opportunistic pathogens during pregnancy, being linked to early-onset disease in premature infants. ${ }^{39,40}$ Women with PTB were six times more likely to increase the frequency of Megasphaera spp. (family Veillonellaceae) through pregnancy. ${ }^{41}$ In addition, autistic patients with gastrointestinal symptoms and liver cirrhosis have distinct (less diverse) gut microbial compositions and lower levels of Veillonellaceae in fecal samples. ${ }^{42,43}$ During pregnancy, Veillonella parvula counts decrease in the mouth cavities of women. ${ }^{44}$ Microbiome changes have been described in the nasal and oral cavities, skin and placenta. ${ }^{1}$ Taken together, these data suggest that EVs derived from Ureaplasma spp. and Megasphaera spp. (family Veillonellaceae) could be associated with infectious diseases in preterm infants.

In addition, we checked the delivery history in non-pregnant women. Some data were missing. The delivery history of the control group was marginally significantly lower in Class III compared with that in Class IV $(P=0.047$, data not shown), and the statistical power was low because of the small number of subjects in the group of non-pregnant women.
Recent evidence has indicated that bacteria-derived EVs are important for host-microbe communication. In the present study, we targeted the metagenome, which was isolated from EVs in the urine, to evaluate the association between bacteriaderived EVs and pregnancy, based on the data that bacteriaderived EVs are excreted via the urinary tract. This study showed that Bacillus spp. EVs were more significantly enriched in pregnant women, whereas Pseudomonas spp. EVs were more dominant in non-pregnant women. The study has certain limitations, including its retrospectively cross-sectional study design. Despite the limitations, the present data will provide insight into understanding the physiology of pregnancy and also reveal diagnostic or therapeutic markers for the prevention of non-pregnancy and/or preterm delivery.

\section{CONFLICT OF INTEREST}

The authors declare no conflict of interest.

\section{ACKNOWLEDGEMENTS}

This study was funded by grants to YJK from the Korea Health Technology R\&D Project through the Korea Health Industry Development Institute (KHIDI), which was funded by the Ministry of Health and Welfare, Republic of Korea (grant number: HI14C0306), to YKK from the Korea Ministry of Health and Welfare, Republic of Korea (HI 13C 0040-010013) and to MR from the National Research Foundation of Korea funded by the Ministry of Science, ICT and Future Planning (NRF-2014R1A1A1005144).

1 Prince AL, Antony KM, Ma J, Aagaard KM. The microbiome and development: a mother's perspective. Semin Reprod Med 2014; 32: 14-22.

2 Lawn JE, Kinney MV, Belizan JM, Mason EM, McDougall L, Larson J et al. Born too soon: accelerating actions for prevention and care of 15 million newborns born too soon. Reprod Health 2013; 10: S6.

3 Menon R. Spontaneous preterm birth, a clinical dilemma: etiologic, pathophysiologic and genetic heterogeneities and racial disparity. Acta Obstet Gynecol Scand 2008; 87: 590-600.

4 Goldenberg RL, Hauth JC, Andrews WW. Intrauterine infection and preterm delivery. N Engl J Med 2000; 342: 1500-1507.

5 Zhou X, Brotman RM, Gajer P, Abdo Z, Schuette U, Ma S et al. Recent advances in understanding the microbiology of the female reproductive tract and the causes of premature birth. Infect Dis Obstet Gynecol (e-pub ahead of print 9 December 2010; doi: 10.1155/2010/737425).

6 Zolotor AJ, Carlough MC. Update on prenatal care. Am Fam Physician 2014; 89: 199-208.

7 Schnarr J, Smaill F. Asymptomatic bacteriuria and symptomatic urinary tract infections in pregnancy. Eur J Clin Invest 2008; 38: 50-57.

8 Kim OY, Hong BS, Park KS, Yoon YJ, Choi SJ, Lee WH et al. Immunization with Escherichia coli outer membrane vesicles protects bacteria-induced lethality via Th1 and Th17 cell responses. J Immunol 2013; 190: 4092-4102.

9 Zhou L, Srisatjaluk R, Justus DE, Doyle RJ. On the origin of membrane vesicles in Gram-negative bacteria. FEMS Microbiol Lett 1998; 163: 223-228.

10 Lee EY, Choi DY, Kim DK, Kim JW, Park JO, Kim S et al. Gram-positive bacteria produce membrane vesicles: proteomics-based characterization of Staphylococcus aureus-derived membrane vesicles. Proteomics 2009; 9: 5425-5436.

$11 \mathrm{Kim}$ MR, Hong SW, Choi EB, Lee WH, Kim YS, Jeon SG et al. Staphylococcus aureus-derived extracellular vesicles induce neutrophilic pulmonary inflammation via both Th1 and Th17 cell responses. Allergy 2012; 67: 1271-1281. 
12 Jang SC, Kim SR, Yoon YJ, Park KS, Kim JH, Lee J et al. In vivo kinetic biodistribution of nano-sized outer membrane vesicles derived from bacteria. Small 2015; 11: 456-461.

13 Keller S, Ridinger J, Rupp AK, Janssen JW, Altevogt P. Body fluid derived exosomes as a novel template for clinical diagnostics. J Trans/ Med 2011; 9: 86.

14 Yanez-Mo M, Siljander PR, Andreu Z, Zavec AB, Borras FE, Buzas El et al. Biological properties of extracellular vesicles and their physiological functions. J Extracell Vesicles 2015; 4: 27066.

15 Aagaard K, Riehle K, Ma J, Segata N, Mistretta TA, Coarfa C et al. A metagenomic approach to characterization of the vaginal microbiome signature in pregnancy. PLOS ONE 2012; 7: e36466.

16 Edwards RA, Rodriguez-Brito B, Wegley L, Haynes M, Breitbart M, Peterson DM et al. Using pyrosequencing to shed light on deep mine microbial ecology. BMC Genomics 2006; 7: 57.

17 Romero R, Espinoza J, Rogers WT, Moser A, Nien JK, Kusanovic JP et al. Proteomic analysis of amniotic fluid to identify women with preterm labor and intra-amniotic inflammation/infection: the use of a novel computational method to analyze mass spectrometric profiling. J Matern Fetal Neonatal Med 2008; 21: 367-388.

18 Yoon BH, Romero R, Kim CJ, Jun JK, Gomez R, Choi JH et al. Amniotic fluid interleukin-6: a sensitive test for antenatal diagnosis of acute inflammatory lesions of preterm placenta and prediction of perinatal morbidity. Am J Obstet Gynecol 1995; 172: 960-970.

19 Gilbert NM, O'Brien VP, Hultgren S, Macones G, Lewis WG, Lewis AL. Urinary tract infection as a preventable cause of pregnancy complications: opportunities, challenges, and a global call to action. Glob Adv Health Med 2013; 2: 59-69.

20 Lee EY, Bang JY, Park GW, Choi DS, Kang JS, Kim HJ et al. Global proteomic profiling of native outer membrane vesicles derived from Escherichia coli. Proteomics 2007; 7: 3143-3153.

21 Edgar RC. Search and clustering orders of magnitude faster than BLAST. Bioinformatics 2010; 26: 2460-2461.

22 Lozupone C, Hamady M, Knight R. UniFrac-an online tool for comparing microbial community diversity in a phylogenetic context. BMC Bioinform 2006; 7: 371.

23 Caporaso JG, Kuczynski J, Stombaugh J, Bittinger K, Bushman FD, Costello EK et al. QIIME allows analysis of high-throughput community sequencing data. Nat Methods 2010; 7: 335-336.

24 Human Microbiome Project Consortium. A framework for human microbiome research. Nature 2012; 486: 215-221.

25 Human Microbiome Project Consortium. Structure, function and diversity of the healthy human microbiome. Nature 2012; 486: 207-214.

26 Andaloussi SEL, Mager I, Breakefield XO, Wood MJ. Extracellular vesicles: biology and emerging therapeutic opportunities. Nat Rev Drug Discov 2013; 12: 347-357.

27 Eisen JA. Environmental shotgun sequencing: its potential and challenges for studying the hidden world of microbes. PLoS Biol 2007; 5: e82.

28 Poretsky R, Rodriguez RL, Luo C, Tsementzi D, Konstantinidis KT. Strengths and limitations of $16 \mathrm{~S}$ rRNA gene amplicon sequencing in revealing temporal microbial community dynamics. PLOS ONE 2014; 9: e93827.

29 Romano-Keeler J, Weitkamp JH. Maternal influences on fetal microbial colonization and immune development. Pediatr Res 2015; 77: 189-195.

30 Kehinde A, Adedapo K, Aimakhu C, Odukogbe AT, Olayemi O, Salako B. Urinary pathogens and drug susceptibility patterns of urinary tract infections among antenatal clinic attendees in Ibadan, Nigeria. J Obstet Gynaecol Res 2012; 38: 280-284.
31 Stein T. Bacillus subtilis antibiotics: structures, syntheses and specific functions. Mol Microbiol 2005; 56: 845-857.

32 Eom JS, Lee SY, Choi HS. Bacillus subtilis HJ18-4 from traditional fermented soybean food inhibits Bacillus cereus growth and toxinrelated genes. J Food Sci 2014; 79: M2279-M2287.

33 Casetta A, Audibert F, Brivet F, Boutros N, Boithias C, Lebrun L. Emergence of nosocomial Pseudomonas aeruginosa colonization/infection in pregnant women with preterm premature rupture of membranes and in their neonates. J Hosp Infect 2003; 54: 158-160.

34 Dosiou C, Giudice LC. Natural killer cells in pregnancy and recurrent pregnancy loss: endocrine and immunologic perspectives. Endocr Rev 2005; 26: 44-62.

35 Brazao V, Kuehn CC, Santos CD, Costa CM, Junior JC, Carraro-Abrahao AA. Endocrine and immune system interactions during pregnancy. Immunobiology 2015; 220: 42-47.

36 Bieri RA, Adriaens L, Sporri S, Lang NP, Persson GR. Gingival fluid cytokine expression and subgingival bacterial counts during pregnancy and postpartum: a case series. Clin Oral Invest 2013; 17: 19-28.

37 Basso B, Gimenez F, Lopez C. IL-1beta, IL-6 and IL-8 levels in gyneco-obstetric infections. Infect Dis Obstet Gynecol 2005; 13: 207-211.

38 Romero R, Miranda J, Chaemsaithong P, Chaiworapongsa T, Kusanovic JP, Dong $Z$ et al. Sterile and microbial-associated intra-amniotic inflammation in preterm prelabor rupture of membranes. J Matern Fetal Neonatal Med 2015; 28: 1394-1409.

39 Cassell GH, Waites KB, Watson HL, Crouse DT, Harasawa R. Ureaplasma urealyticum intrauterine infection: role in prematurity and disease in newborns. Clin Microbiol Rev 1993; 6: 69-87.

40 Han YW, Shen T, Chung P, Buhimschi IA, Buhimschi CS. Uncultivated bacteria as etiologic agents of intra-amniotic inflammation leading to preterm birth. J Clin Microbiol 2009; 47: 38-47.

41 Nelson DB, Hanlon A, Nachamkin I, Haggerty C, Mastrogiannis DS, Liu C et al. Early pregnancy changes in bacterial vaginosis-associated bacteria and preterm delivery. Paediatr Perinat Epidemiol 2014; 28: 88-96.

42 Chen Y, Yang F, Lu H, Wang B, Lei D, Wang Y et al. Characterization of fecal microbial communities in patients with liver cirrhosis. Hepatology 2011; 54: 562-572.

43 Kang DW, Park JG, Ilhan ZE, Wallstrom G, Labaer J, Adams JB et al. Reduced incidence of Prevotella and other fermenters in intestinal microflora of autistic children. PLOS ONE 2013; 8: e68322.

44 Adriaens LM, Alessandri R, Sporri S, Lang NP, Persson GR. Does pregnancy have an impact on the subgingival microbiota? J Periodontol 2009; 80: $72-81$.

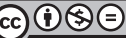

This work is licensed under a Creative Commons Attribution-NonCommercial-NoDerivs 4.0 International License. The images or other third party material in this article are included in the article's Creative Commons license, unless indicated otherwise in the credit line; if the material is not included under the Creative Commons license, users will need to obtain permission from the license holder to reproduce the material. To view a copy of this license, visit http://creativecommons.org/licenses/by-nc-nd/4.0/

Supplementary Information accompanies the paper on Experimental \& Molecular Medicine website (http://www.nature.com/emm) 